\title{
The repeatability of bilateral diffusion tensor imaging (DTI) in the upper leg muscles of healthy adults
}

\author{
Jithsa R. Monte ${ }^{1} \mathbb{D} \cdot$ Melissa T. Hooijmans $^{2} \cdot$ Martijn Froeling $^{3} \cdot$ Jos Oudeman $^{1} \cdot$ Johannes L. Tol $^{4} \cdot$ Mario Maas $^{1}$. \\ Gustav J. Strijkers ${ }^{2}$. Aart J. Nederveen ${ }^{1}$
}

Received: 15 May 2019 / Revised: 10 July 2019 / Accepted: 29 July 2019/Published online: 8 November 2019

(C) The Author(s) 2019

\begin{abstract}
Objectives Assessment of the repeatability of diffusion parameter estimations in the upper leg muscles of healthy adults over the time course of 2 weeks, from a simultaneous bilateral upper leg DTI measurement.

Methods SE-EPI DTI datasets were acquired at $3 \mathrm{~T}$ in the upper legs of 15 active adults at a time interval of 2 weeks. ROIs were manually drawn for four quadriceps and three hamstring muscles of both legs. The following DTI parameters were analyzed: $1 \mathrm{st}$, 2nd, and 3rd eigenvalue ( $\lambda_{1}, \lambda_{2}$, and $\left.\lambda_{3}\right)$, mean diffusivity (MD), and fractional anisotropy (FA). DTI parameters per muscle were calculated with and without intravoxel incoherent motion (IVIM) correction together with SNR levels per muscle. Bland-Altman plots and within-subject coefficient of variation (wsCV) were calculated. Left-right differences between muscles were assessed. Results The Bland-Altman analysis showed good repeatability of all DTI parameters except FA for both the IVIM-corrected and standard data. wsCV values show that MD has the highest repeatability (4.5\% IVIM; $5.6 \%$ standard), followed by $\lambda_{2}$ (4.9\% IVIM; $5.5 \%$ standard), $\lambda_{1}$ (5.3\% IVIM; $7.5 \%$ standard), and $\lambda_{3}$ (5.7\% IVIM; $5.7 \%$ standard). wsCV values of FA were $15.2 \%$ for the IVIM-corrected data and $13.9 \%$ for the standard analysis. The SNR (41.8 \pm 16.0 right leg, $41.7 \pm 17.1$ left leg) and wsCV values were similar for the left and right leg and no left-right bias was detected.

Conclusions Repeatability was good for standard DTI data and slightly better for IVIM-corrected DTI data. Our protocol is suitable for DTI of the upper legs with overall good SNR.

Key Points

- The presented DTI protocol is repeatable and therefore suitable for bilateral DT imaging of the upper legs.

- Additional $\mathrm{BI}^{+}$calibrations improve SNR and repeatability.

- Correcting for perfusion effects improves repeatability.
\end{abstract}

Keywords Diffusion tensor imaging · Magnetic resonance imaging · Hamstring muscles · Quadriceps muscle

Electronic supplementary material The online version of this article (https://doi.org/10.1007/s00330-019-06403-5) contains supplementary material, which is available to authorized users.

Jithsa R. Monte

j.r.monte@amc.uva.nl

1 Amsterdam UMC, Radiology \& Nuclear Medicine, University of Amsterdam, Meibergdreef 9, Amsterdam, The Netherlands

2 Amsterdam UMC, Biomedical Engineering and Physics, University of Amsterdam, Meibergdreef 9, Amsterdam, The Netherlands

3 Department of Radiology, University Medical Center Utrecht, Utrecht, The Netherlands

4 Amsterdam UMC, Department of Orthopaedic Surgery, University of Amsterdam, Meibergdreef 9, Amsterdam, The Netherlands

\begin{tabular}{ll}
\multicolumn{2}{l}{ Abbreviations } \\
BFL & Left biceps femoris long head \\
BFR & Right biceps femoris long head \\
IVIM & Intravoxel incoherent motion \\
MD & Mean diffusivity \\
RFL & Left rectus femoris \\
RFR & Right rectus femoris \\
ROI & Region of interest \\
SML & Left semimembranosus \\
SMR & Right semimembranosus \\
SNR & Signal-to-noise ratio \\
STL & Left semitendinosus \\
STR & Right semitendinosus \\
VIL & Left vastus intermedius \\
VIR & Right vastus intermedius
\end{tabular}

\section{Abbreviations}

BFR Right biceps femoris long head

IVIM Intravoxel incoherent motion

MD Mean diffusivity

RFL Left rectus femoris

RFR Right rectus femoris

ROI Region of interest

SML Left semimembranosus

SMR Right semimembranosus

SNR Signal-to-noise ratio

STL Left semitendinosus

STR Right semitendinosus

VIR Right vastus intermedius 
VLL Left vastus lateralis

VLR Right vastus lateralis

VML Left vastus medialis

VMR Right vastus medialis

$\lambda_{1} \quad$ First Eigenvalue

$\lambda_{2} \quad$ Second eigenvalue

$\lambda_{3}$ Third eigenvalue motion (IVIM) model improves DTI parameter estimations and potential repeatability [20].

The combined main aims of this study were therefore to assess the repeatability of diffusion parameter estimations in the upper leg muscles of healthy active from a simultaneous bilateral upper leg DTI measurement, with and without IVIM correction. Furthermore, we also considered the benefits of additional $\mathrm{B}^{+}$calibrations. We hypothesized that additional prescans prior to DTI could improve $\mathrm{B} 1^{+}$homogeneity [21].

\section{Methods and materials}

Muscle injuries are the most prevalent injuries in elite and recreational sports [1]. Although considerable progress has been made, effective treatment and rehabilitation of muscle injury, particularly those affecting leg muscles, remain challenging as demonstrated by the high recurrence incidence after acute hamstring injury $[2,3]$. For diagnostic purposes, conventional T2-weighted fat-suppressed MRI is commonly used [4]. Unfortunately, a major drawback is that hyperintensity - a characteristic of the injury - remains visible in these images long after all clinical symptoms have cleared and the athlete has resumed sports activity [5]. This implies that T2-weighted imaging with fat suppression is insufficient for evaluating muscle recovery during rehabilitation and for assessing return to play (RTP) [6-8]. More sensitive and quantitative methods are therefore highly desired.

Over the past decades, diffusion tensor imaging (DTI) MRI emerged as a promising technique to evaluate muscle tissue repair [9]. Previous work showed that DTI facilitated detection of structural changes in muscle beyond conventional T2weighted MRI [10]. Also, research performed in injured athletes showed that DTI has the potential for the assessment of muscle injuries [11, 12].

Despite its promise for diagnosis and follow-up of sportsrelated muscle injury, repeatability of the muscle DTI method needs to be established and a couple of experimental hurdles need to be overcome. First, most DTI studies with 3-T MRI systems targeting the upper legs have been performed in a single left or right leg, because imaging of both legs resulted in varying image quality stemming from B1 inhomogeneities [13]. However, a bilateral assessment is important as the contralateral leg serves as an internal reference to control for between-subject variations in DTI indices introduced by differences in age, gender, and activity level [14, 15]. Bilateral imaging therefore will significantly increase the discriminative power of the technique, provided DTI of both legs simultaneously provides sufficient image quality to derive accurate and repeatable quantitative diffusion indices $[16,17]$. Secondly, signal attenuation originating from blood flow in the microvascular network (perfusion) can be a confounding factor $[18,19]$. Previous research has shown that modeling the signal attenuation with a so-called intravoxel incoherent
This study was waived by the local IRB as no patients participated. We obtained written informed consent from all volunteers prior to the study.

\section{Subjects}

Eighteen healthy subjects, 16 males and 2 females, mean age $28.4 \pm 5.2$ years (range $22-40$ years) with no recorded upper leg injury in the half-year prior to this study were recruited. Inclusion criteria were the following: healthy, at least 18 years old, and active in sports. Subjects engaged in sports activities on average 2.9 times per week and were instructed to continue with their sport/exercise regimen. Exclusion criteria were the following: MRI contra-indications, injury of one of the leg muscles seen on MRI, or unable to fully complete both scanning sessions. The protocol was performed twice with a 2week interval (average 14 days, range 13-15 days). One subject was excluded based on a muscle injury detected at the first scanning session and two subjects had incomplete datasets due to scheduling conflicts. Thus, 15 subjects (13 males, 2 females) were finally included.

\section{MRI}

MRI was acquired with a 3-T Philips Ingenia MRI scanner (Philips Healthcare) using a 16-channel receive array coil placed on top of the legs in combination with 12 receive coils in the patient table. Subjects were placed supine and feet first in the scanner. Dielectric pads were placed on top of the left upper leg and under the right upper leg [13]. These pads served to reduce RF inhomogeneity by augmenting the applied $\mathrm{B} 1^{+}$field. The geometrical planning for the second session was carefully matched to one of the first session using anatomical landmarks and saved images of the slice planning.

The MRI examination consisted of four sequences:

1. Axial DTI sequence with multiple $b$ values for the purpose of IVIM correction [20];

2. Axial T2-weighted sequence for anatomical reference; 
3. Axial PD-weighted DIXON sequence for the purpose of segmentation;

4. Coronal T2-weighted sequence to assess potential muscle injury (exclusion criterion).

Total scanning time was $25 \mathrm{~min}$. Table 1 lists detailed scanning parameters. Additional DTI parameters: sequence $=$ diffusion-weighted spin-echo - echo-planar-imaging (SE-EPI), fat suppression $=$ SPAIR (spectral attenuated inversion recovery), no slice gap, fold-over direction $=$ anterior-posterior, $b$ values (no. gradient orientations) $=0(1 \times), 1(8 \times), 5(3 \times), 10$ $(3 \times), 20(3 \times), 50(3 \times), 100(3 \times), 200(10 \times), 400(10 \times), 600$ $(12 \times) \mathrm{s} / \mathrm{mm}^{2}$ (10 b values, 56 gradient directions), scan duration $11 \mathrm{~m} 8 \mathrm{~s}$, receiver bandwidth $27.9 \mathrm{~Hz} /$ pixel, echo times of the axial PD-weighted DIXON sequence: $1.35 \mathrm{~ms}, 2.45 \mathrm{~ms}$, $3.55 \mathrm{~ms}, 4.65 \mathrm{~ms}, 5.75 \mathrm{~ms}$, and $6.85 \mathrm{~ms}$.

\section{IVIM correction}

For the IVIM-corrected data, first, the full IVIM model was fitted to the average signal per $b$ value using NLS model (initialization parameters, $\mathrm{S} 0=1 ; f=0.05 ; D=0.003 ; D \mathrm{p}=$ 0.1 ). The isotropic perfusion component of the signal is calculated and subtracted from the signal of all diffusion directions and $b$ values. The perfusion-corrected signal of all $b$ values and directions was then used for tensor estimation using an iterative weighted linear least squares (iWLLS) algorithm [20].

\section{$\mathrm{B1}^{+}$calibration}

The 30 -s prescans included a $\mathrm{B}^{+}$calibration scan, a SENSE reference scan, and a coil survey scan. The prescans are proprietary Philips technology and are currently performed automatically on Philips Ingenia Systems (since release R5.3.0) at the beginning of the protocol and any time when a substantial change in the field of view or movement of the scanner table occurs. We performed additional prescans prior to the DTI sequence for 5 subjects.

\section{Analysis}

DTI data were processed using the custom-built software DTITools (https://github.com/mfroeling/DTITools) for Wolfram Mathematica 11.3. First, the diffusion-weighted images were pre-processed using a principle component analysis (PCA) noise suppression algorithm [22]. Subsequently, the diffusion-weighted images were registered to the T2-weighted axial images, using rigid and bspline transformations [23] to correct for subject motion and image deformations due to eddy currents. An iterative weighted linear least squares algorithm [24] was used for the estimation of $\lambda_{1}, \lambda_{2}$, and $\lambda_{3}$ from which mean diffusivity (MD) and fractional anisotropy (FA) were calculated. Diffusion attenuation was either modeled using a single exponential decay model-this will be referred to as "standard" - or by IVIM modeling to correct the diffusion parameters for the effects of pseudo-diffusion (perfusion) [20]. The latter will be referred to as "IVIM-corrected." Signal-to-noise ratio (SNR) per muscle was estimated to assess DTI quality. The standard deviation $(\sigma)$ of the noise was mapped through the PCA denoising process [22]. This $\sigma$ map was used for the SNR estimations [25]. SNR was defined as the mean of the signal in a muscle ROI divided by $\sigma$. In case of $\mathrm{SNR}<20$, this muscle was excluded from further analysis [16].

The analysis was done for the following muscles of the right $(\mathrm{R})$ and left $(\mathrm{L})$ legs: rectus femoris $(\mathrm{RF})$, vastus medialis (VM), vastus intermedius (VI), vastus lateralis (VL), semitendinosus (ST), biceps femoris long head (BF), and semimembranosus (SM). Manual segmentation of the muscles was performed in ITK-snap [26] (www.itksnap.org) in the out-of-phase PD-weighted DIXON (axial) images. The segmentation was performed slice by slice, carefully excluding subcutaneous fat (Fig. 1). The segmentations were imported in Mathematica as masks, eroded 1 pixel, and rescaled to the resolution of the DTI images for the quantification of the DTI parameters and SNR per muscle. The mean value of the estimated DTI indices was calculated over the 20 middle slices. The injury in our clinical study is always placed in the middle of the field of view planning. Hence, we are

Table 1 MRI scan parameters

\begin{tabular}{llllllll}
\hline Sequence & OR & FOV $\left(\mathrm{mm}^{2}\right)$ & VOX $\left(\mathrm{mm}^{3}\right)$ & TR/TE $(\mathrm{ms})$ & NSA & Echo & Slices \\
\hline DTI (SE-EPI) & AX & $480 \times 252$ & $3 \times 3 \times 5$ & $5914 / 54.56$ & 2 & 1 & 40 \\
T2 (MS_TSE) & AX & $480 \times 252$ & $1.5 \times 1.5 \times 2.5$ & $2000 / 70$ & 1 & 1 & 80 \\
PD DIXON (FFE) & AX & $480 \times 252$ & $1.5 \times 1.5 \times 2.5$ & $500 / 1.35$ & 3 & 6 & 80 \\
T2 (MS_TSE) & COR & $450 \times 450$ & $0.55 \times 0.66 \times 4$ & $2000 / 60$ & 1 & 1 & 90 \\
\hline
\end{tabular}

$A X$, axial; $C O R$, coronal; FOV, field of view; VOX, voxel dimensions; NSA, number of signal averages; $T R$, repetition time; $T E$, echo time; $P I F$, parallel imaging factor; $O R$, orientation 
Fig. 1 a Representative axial proton density-weighted MRI with overlaid muscle segmentations in color. b 3D volume rendering of segmented muscles in the center 20 slices of the upper leg

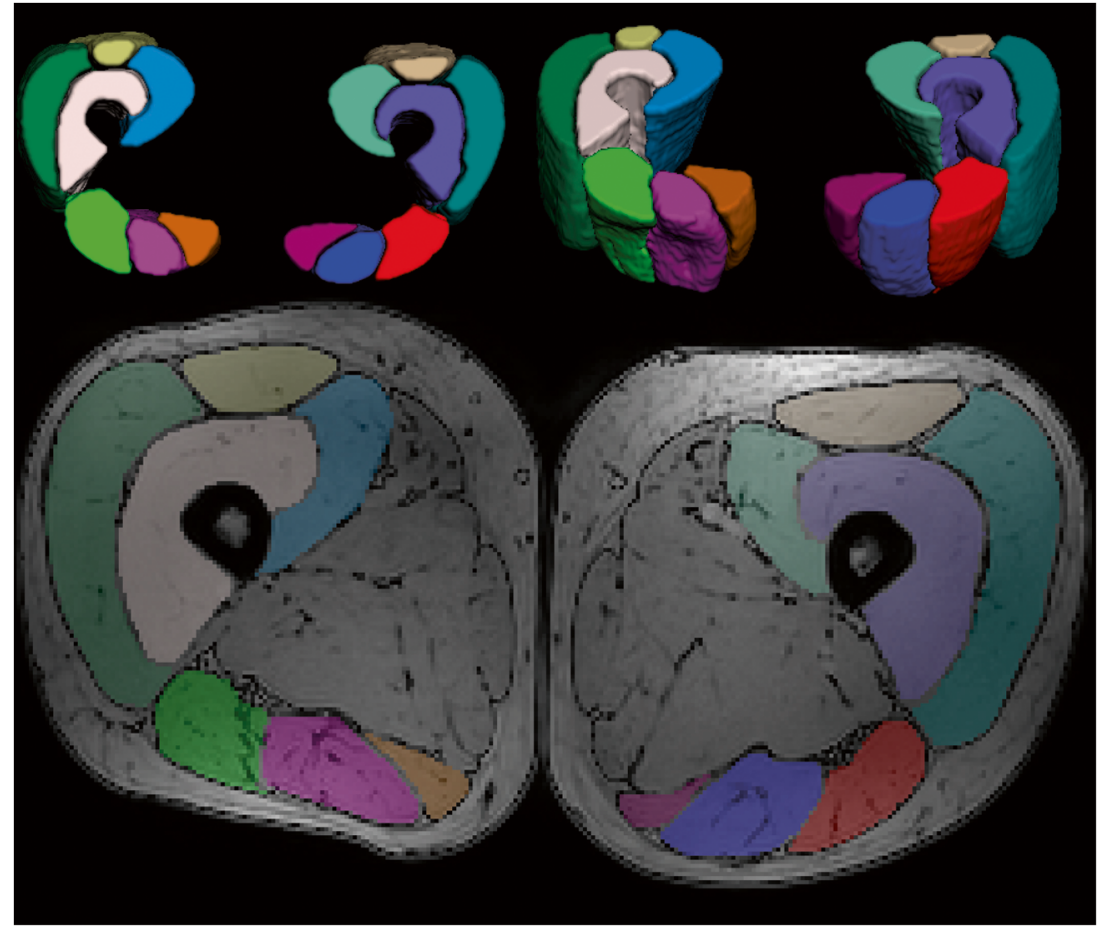

interested in the quality and repeatability of the 20 middle slices of the sequence.

\section{Statistical analysis}

The repeatability of the two measurements was assessed with Bland-Altman plots and calculations of the within-subject coefficient of variation (wsCV), which was calculated as the ratio of the standard deviation of the paired differences to the mean.

The statistical analysis was performed for the standard and IVIM-corrected data, and separately for the right and left legs. Paired samples $t$ tests were done to compare the wsCV values per DTI parameter for the IVIM-corrected data with standard data. The same approach was used to compare wsCV values per DTI parameter between the subgroups of 5 and 10 subjects for the IVIM-corrected data. Significance was set at $p<0.05$. The minimal detectable difference, defined as $1.96 \times$ standard deviation of the paired differences, was calculated per muscle per DTI parameter.

\section{Results}

\section{Data quality}

SNR calculations for 420 muscles ( 15 participants $\times 7$ muscles per leg $\times 2$ legs $\times 2$ time points) resulted in the exclusion of $50(11.9 \%)$ muscles due to insufficient SNR $(<20)$ leaving
185 muscle pairs (370) for further analysis. Mean SNR for all included muscles was $44.5 \pm 8.9$.

Mean SNR of all muscles was $41.8 \pm 16.0$ for the right and $41.7 \pm 17.1$ for the left leg. An overview of the numbers of included and excluded muscles per subject, as well as mean SNR values, is given in Supplemental Tables 1 and 2.

The visual assessment showed that, despite fat suppression and $\mathrm{B} 1^{+}$shimming, chemical shift artifacts and shading artifacts $\left(\mathrm{B} 1^{+}\right.$-field inhomogeneities) were present in a majority of the images (Supplemental Figure 1). The artifacts were mostly present in the RF muscle, causing an overall SNR drop in the quadriceps muscles compared with that in the hamstring muscles. The shading artifacts were primarily present in the upper quadrant of the left leg (quadriceps) and in the lower quadrant of the right leg (hamstrings) and were most apparent in the left quadriceps. These artifacts caused SNR differences between left and right with higher SNR in the left hamstrings and quadriceps (Supplemental Table 2).

Despite these regional artifacts, all datasets were of sufficient quality for further processing. Figure 2 provides an overview of the images on the 1st time point for all 15 subjects. Figure 3 shows images from two time points for the dataset with the lowest SNR and a representative dataset with high SNR. For both the lower and higher SNR datasets, the anatomical registration was good.

\section{DTI parameters}

Average values and standard deviation of the individual DTI parameters per muscle are reported in Table 2. The mean 


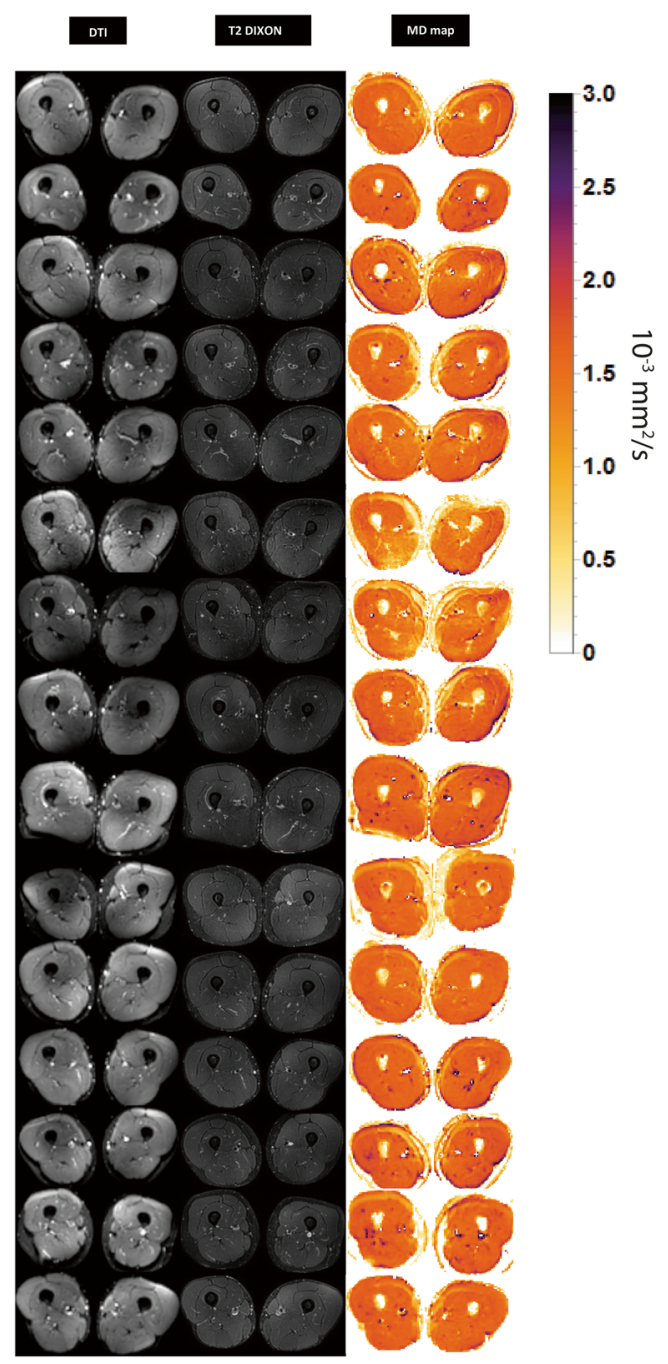

Fig. 2 Representative axial slice of the DTI ( $b$ value $\left.0 \mathrm{~s} / \mathrm{mm}^{2}\right)$ images, T2-weighted images, and MD maps for all 15 subjects at the first time point

values for all IVIM-corrected DTI parameters except FA were lower than the mean values of the standard DTI parameters due to the absence of perfusion contamination. The BlandAltman analysis showed good agreement between time points 1 and 2 for all DTI parameters, except FA for both standard and IVIM-corrected data (Figs. 4 and 5). wsCV values varied between 2.2 and $37.7 \%$ for IVIM-corrected DTI data and between 2.9 and $32.3 \%$ for the standard DTI data (Table 3).

The MD had the highest repeatability (4.5\% IVIM; $5.6 \%$ standard), followed by $\lambda_{2}$ (4.9\% IVIM; $5.5 \%$ standard), $\lambda_{1}$ (5.3\% IVIM; $7.5 \%$ standard), and $\lambda_{3}$ (5.7\% IVIM; $5.7 \%$ standard). FA had the lowest repeatability of all DTI parameters, $15.2 \%$ for the IVIM-corrected data and $13.9 \%$ for the standard analysis. For an overview of the wsCV and its standard deviation (SD) per DTI parameter, calculated as the mean of all muscles per DTI parameter, see Table 4 . The mean wsCV values over all muscles of $\lambda_{1}(p=0.003)$ and $\operatorname{MD}(p=0.02)$ were significantly lower for the IVIM-corrected data. The wsCV values of $\lambda_{2}(p=0.14), \lambda_{3}(p=0.96)$, and FA ( $p=$ 0.05 ) for the IVIM-corrected data were also lower but not significantly. The mean wsCV (IVIM-corrected data) was comparable for the left and right legs (Table 3).

The IVIM-corrected DTI data showed slightly lower values for the minimal detectable difference compared with the standard analysis. This was the case for all DTI parameters except FA (Supplemental Table 5).

\section{Additional $\mathrm{B} 1^{+}$calibration}

For this group, 2 muscles out of 140 muscles ( 5 subjects $\times 7$ muscles $\times 2$ legs $\times 2$ time points) were excluded from the analysis due to insufficient SNR $(<20)$. Mean SNR (all muscles) was $50.0 \pm 12.2$ for the 5 subjects with additional calibration vs. $37.6 \pm 16.9$ for the 10 standard subjects. The mean wsCV values per DTI parameter for the IVIM-corrected data were lower for these 5 subjects compared with those for the 10 subjects with the regular protocol (Supplemental Table 3). This was significant for $\lambda_{2}$ $(p=0.04), \lambda_{3}(p=0.03)$, and MD $(p=0.04)$, but not for $\lambda_{1}(p=0.06)$ and FA $(p=0.1)$.

\section{SNR in relation to DTI indices}

The IVIM-corrected MD and FA were plotted against the SNR for 2 hamstring (BF and ST) and 2 quadriceps (VM and VI) muscles of the left and right legs (Fig. 6) in order to detect potential SNR bias. An underestimation of MD was present in the lower SNR range $($ SNR $<20)$ but no overestimation of FA.

\section{Discussion}

Our research showed good repeatability of the DTI parameters in the upper leg muscles using a clinically applicable protocol suited for imaging of both legs simultaneously. The repeatability became even slightly better after IVIM correction for perfusion. Furthermore, performing an additional $\mathrm{B}^{+}$calibration improved SNR and repeatability of the DTI parameter estimations. Lastly, we found that the data quality (SNR) and repeatability (wsCV) of the DTI parameters were similar for the left and right legs.

\section{Data quality}

The overall quality of the images was visually judged good. However, chemical shift artifacts by residual unsuppressed fat and shading due to $\mathrm{B} 1^{+}$field inhomogeneities were regionally observed. The direction of the fat shift was chosen from anterior to posterior ensuring that residual fat signal would not 


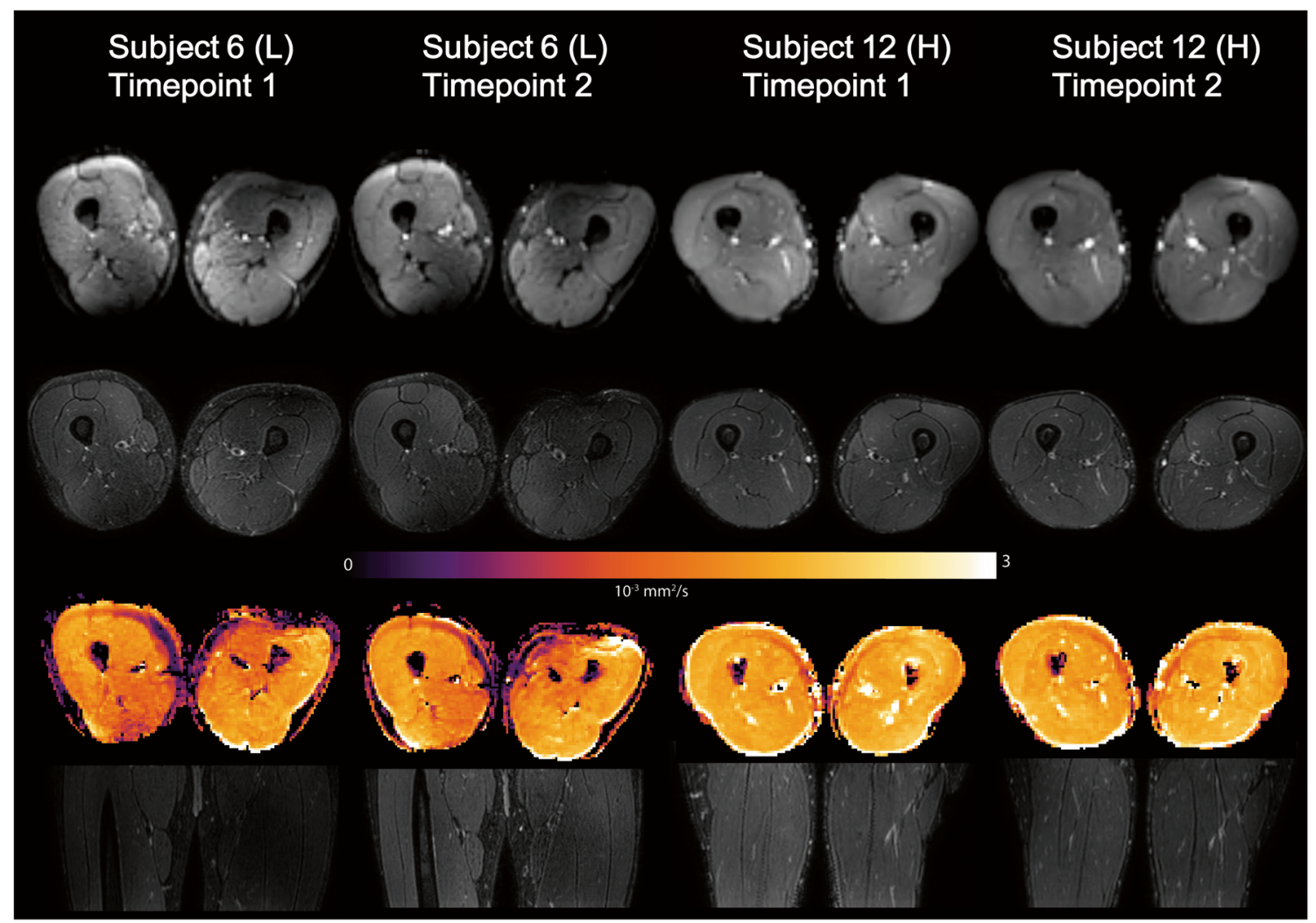

Fig. 3 First and second time point scans of two subjects with (L) the lowest SNR and (H) typical high SNR. From top to bottom: axial DTI ( $b$ value $\left.=0 \mathrm{~s} / \mathrm{mm}^{2}\right)$, T2-weighted images, MD map, coronal T2- weighted images. Mean SNR subject 6: time point 1, $24.8 \pm 14.4$; time point 2, 27.9 \pm 13.4 . Mean SNR subject 12: time point 1, $47.6 \pm 11.5$; time point $2,54.2 \pm 14$

due to B0-field inhomogeneities. A research pre-pulse (saturation pulse) did improve suppression of the lipid olefinic resonance, but at the cost of decreased overall data quality shift into the commonly injured hamstring muscles. However, this resulted in some fat artifacts in the quadriceps muscles. The residual fat signal is caused by suboptimal fat suppression

Table 2 Mean DTI indices $\left(10^{-3} \mathrm{~mm}^{2} / \mathrm{s}\right)$ for all subjects at time point 1

\begin{tabular}{|c|c|c|c|c|c|c|c|c|c|c|}
\hline \multicolumn{6}{|c|}{ Standard } & \multicolumn{5}{|l|}{ IVIM } \\
\hline Muscle & $\begin{array}{l}\lambda_{1} \\
\left(10^{-3} \mathrm{~mm}^{2} / \mathrm{s}\right)\end{array}$ & $\begin{array}{l}\lambda_{2} \\
\left(10^{-3} \mathrm{~mm}^{2} / \mathrm{s}\right)\end{array}$ & $\begin{array}{l}\lambda_{3} \\
\left(10^{-3} \mathrm{~mm}^{2} / \mathrm{s}\right)\end{array}$ & $\begin{array}{l}\mathrm{MD} \\
\left(10^{-3} \mathrm{~mm}^{2} / \mathrm{s}\right)\end{array}$ & $\begin{array}{l}\text { FA } \\
(-)\end{array}$ & $\begin{array}{l}\lambda_{1} \\
\left(10^{-3} \mathrm{~mm}^{2} / \mathrm{s}\right)\end{array}$ & $\begin{array}{l}\lambda_{2} \\
\left(10^{-3} \mathrm{~mm}^{2} / \mathrm{s}\right)\end{array}$ & $\begin{array}{l}\lambda_{3} \\
\left(10^{-3} \mathrm{~mm}^{2} / \mathrm{s}\right)\end{array}$ & $\begin{array}{l}\text { MD } \\
\left(10^{-3} \mathrm{~mm}^{2} / \mathrm{s}\right)\end{array}$ & $\begin{array}{l}\text { FA } \\
(-)\end{array}$ \\
\hline BFL & $2.23 \pm 0.20$ & $1.70 \pm 0.07$ & $1.47 \pm 0.08$ & $1.80 \pm 0.1$ & $0.21 \pm 0.04$ & $2.14 \pm 0.16$ & $1.61 \pm 0.06$ & $1.38 \pm 0.08$ & $1.71 \pm 0.08$ & $0.22 \pm 0.04$ \\
\hline BFR & $2.19 \pm 0.14$ & $1.67 \pm 0.07$ & $1.48 \pm 0.06$ & $1.78 \pm 0.07$ & $0.20 \pm 0.04$ & $2.06 \pm 0.10$ & $1.57 \pm 0.06$ & $1.39 \pm 0.06$ & $1.67 \pm 0.05$ & $0.21 \pm 0.04$ \\
\hline RFL & $1.88 \pm 0.19$ & $1.41 \pm 0.13$ & $1.21 \pm 0.11$ & $1.50 \pm 0.13$ & $0.23 \pm 0.05$ & $1.82 \pm 0.15$ & $1.36 \pm 0.12$ & $1.15 \pm 0.11$ & $1.44 \pm 0.11$ & $0.24 \pm 0.05$ \\
\hline RFR & $1.89 \pm 0.21$ & $1.43 \pm 0.1$ & $1.24 \pm 0.12$ & $1.52 \pm 0.10$ & $0.21 \pm 0.05$ & $1.74 \pm 0.14$ & $1.32 \pm 0.12$ & $1.14 \pm 0.14$ & $1.40 \pm 0.12$ & $0.22 \pm 0.05$ \\
\hline SML & $2.10 \pm 0.16$ & $1.65 \pm 0.11$ & $1.37 \pm 0.15$ & $1.71 \pm 0.13$ & $0.22 \pm 0.04$ & $1.98 \pm 0.14$ & $1.55 \pm 0.11$ & $1.28 \pm 0.16$ & $1.60 \pm 0.13$ & $0.23 \pm 0.04$ \\
\hline SMR & $2.08 \pm 0.12$ & $1.68 \pm 0.1$ & $1.48 \pm 0.08$ & $1.75 \pm 0.10$ & $0.18 \pm 0.01$ & $1.97 \pm 0.09$ & $1.56 \pm 0.07$ & $1.38 \pm 0.08$ & $1.64 \pm 0.08$ & $0.19 \pm 0.02$ \\
\hline STL & $2.22 \pm 0.15$ & $1.66 \pm 0.09$ & $1.41 \pm 0.08$ & $1.76 \pm 0.08$ & $0.23 \pm 0.03$ & $2.14 \pm 0.11$ & $1.60 \pm 0.1$ & $1.34 \pm 0.08$ & $1.69 \pm 0.07$ & $0.24 \pm 0.03$ \\
\hline STR & $2.16 \pm 0.12$ & $1.64 \pm 0.09$ & $1.44 \pm 0.1$ & $1.75 \pm 0.09$ & $0.22 \pm 0.03$ & $2.05 \pm 0.12$ & $1.53 \pm 0.09$ & $1.34 \pm 0.10$ & $1.64 \pm 0.1$ & $0.23 \pm 0.02$ \\
\hline VIL & $2.19 \pm 0.11$ & $1.76 \pm 0.06$ & $1.49 \pm 0.04$ & $1.81 \pm 0.06$ & $0.19 \pm 0.02$ & $2.05 \pm 0.08$ & $1.65 \pm 0.06$ & $1.39 \pm 0.05$ & $1.70 \pm 0.05$ & $0.20 \pm 0.02$ \\
\hline VIR & $2.25 \pm 0.30$ & $1.76 \pm 0.07$ & $1.54 \pm 0.04$ & $1.85 \pm 0.13$ & $0.16 \pm 0.02$ & $1.99 \pm 0.08$ & $1.61 \pm 0.05$ & $1.41 \pm 0.06$ & $1.67 \pm 0.05$ & $0.17 \pm 0.03$ \\
\hline VLL & $2.20 \pm 0.16$ & $1.69 \pm 0.09$ & $1.42 \pm 0.09$ & $1.77 \pm 0.09$ & $0.22 \pm 0.04$ & $2.11 \pm 0.16$ & $1.61 \pm 0.1$ & $1.35 \pm 0.09$ & $1.69 \pm 0.11$ & $0.23 \pm 0.04$ \\
\hline VLR & $2.05 \pm 0.09$ & $1.69 \pm 0.09$ & $1.47 \pm 0.12$ & $1.74 \pm 0.09$ & $0.17 \pm 0.04$ & $1.92 \pm 0.10$ & $1.57 \pm 0.13$ & $1.33 \pm 0.18$ & $1.61 \pm 0.13$ & $0.20 \pm 0.06$ \\
\hline VML & $2.00 \pm 0.09$ & $1.62 \pm 0.07$ & $1.42 \pm 0.06$ & $1.68 \pm 0.07$ & $0.18 \pm 0.01$ & $1.88 \pm 0.08$ & $1.51 \pm 0.06$ & $1.31 \pm 0.06$ & $1.57 \pm 0.06$ & $0.18 \pm 0.02$ \\
\hline VMR & $2.02 \pm 0.11$ & $1.62 \pm 0.06$ & $1.43 \pm 0.06$ & $1.69 \pm 0.07$ & $0.17 \pm 0.02$ & $1.83 \pm 0.09$ & $1.48 \pm 0.05$ & $1.30 \pm 0.06$ & $1.54 \pm 0.06$ & $0.18 \pm 0.02$ \\
\hline
\end{tabular}

DTI indices are given for standard and IVIM modeling of the diffusion attenuation as function of $b$ value. $R F R$, right rectus femoris; $R F L$, left rectus femoris; $V M R$, right vastus medialis; $V M L$, left vastus medialis; VIR, right vastus intermedius; VIL, left vastus intermedius; VLR, right vastus lateralis; $V L L$, left vastus lateralis; $S T R$, right semitendinosus; $S T L$, left semitendinosus; $B F R$, right biceps femoris long head; $B F L$, left biceps femoris long head; $S M R$, right semimembranosus; $S M L$, left semimembranosus 
$\lambda_{1}$

$\lambda_{2}$

$\lambda_{3}$
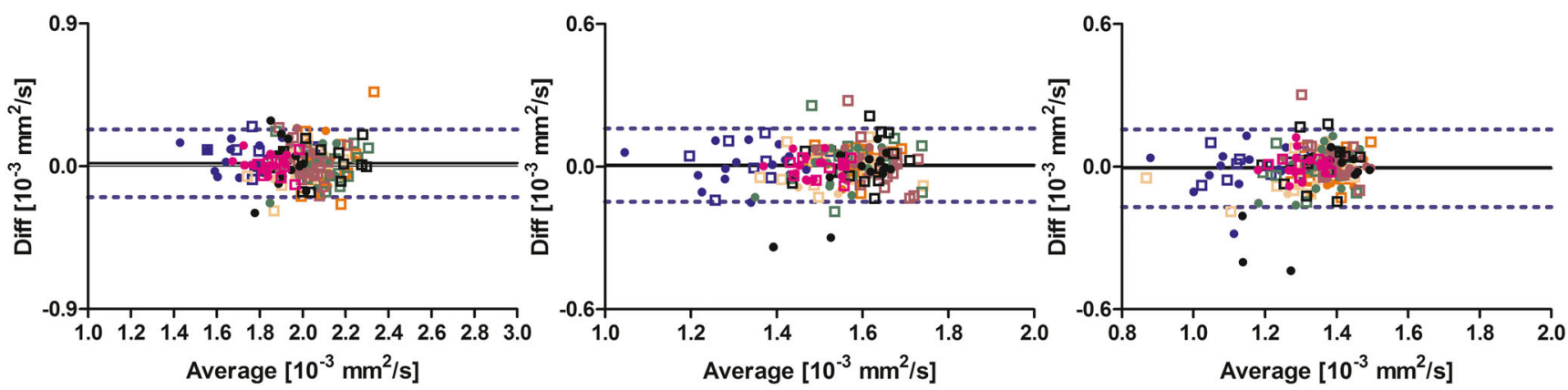

MD

FA

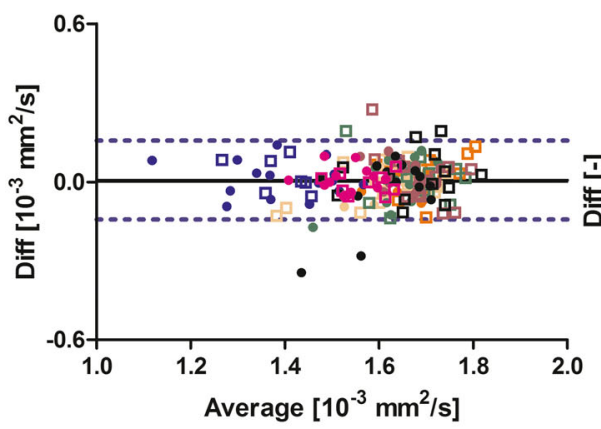

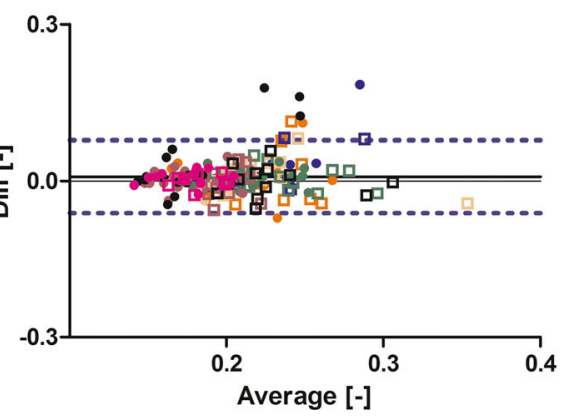

ㅁ Left Biceps Femoris

- Right Biceps Femoris

- Left Rectus Femoris

- Right Rectus Femoris

- Left Semimembranosus

Right Semimembranosus

Left Semitendinosus

Right Semitendinosus

Left Vastus Intermedius

Right Vastus Intermedius

- Left Vastus Lateralis

- Right Vastus Lateralis

- Left Vastus Medialis

- Right Vastus Medialis

Fig. 4 Bland-Altman plots of the IVIM-corrected DTI parameters per muscle. The 95\% confidence interval and the mean of the paired difference are indicated by dashed and solid lines
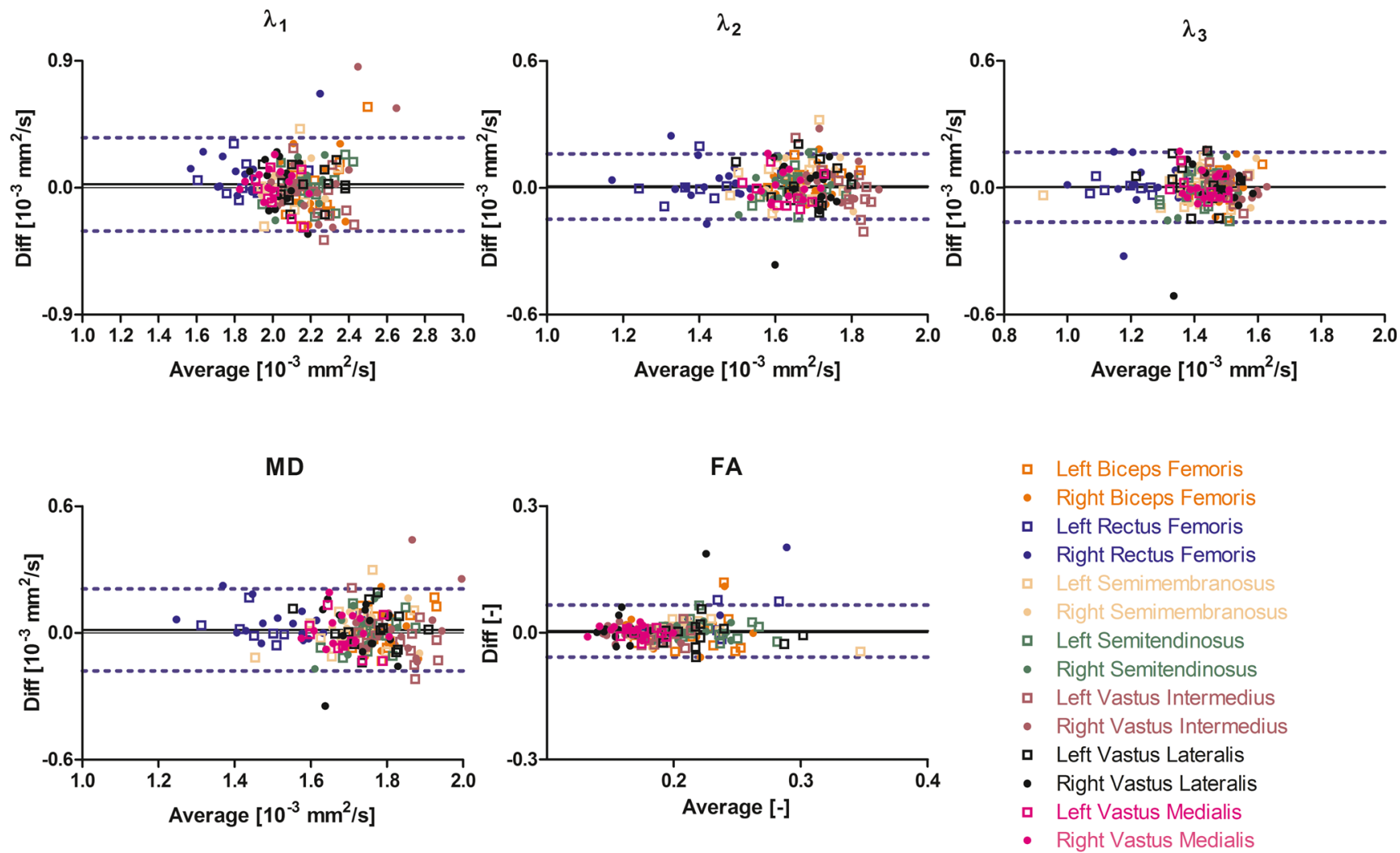

Fig. 5 Bland-Altman plots of the standard DTI parameters per muscle. The $95 \%$ confidence interval and the mean of the paired difference are indicated by dashed and solid lines 
Table 3 wsCV (\%) per muscle for each DTI parameter determined by standard and IVIM modeling

\begin{tabular}{|c|c|c|c|c|c|c|c|c|c|c|}
\hline \multicolumn{6}{|c|}{ Standard } & \multicolumn{5}{|c|}{ IVIM } \\
\hline Muscle & $\lambda_{1} *$ & $\lambda_{2}$ & $\lambda_{3}$ & $\mathrm{MD}^{*}$ & FA & $\lambda_{1} *$ & $\lambda_{2}$ & $\lambda_{3}$ & MD* & FA \\
\hline BFL & 9.2 & 3.4 & 4.4 & 4.8 & 18.7 & 8.0 & 3.4 & 4.9 & 4.2 & 19.7 \\
\hline BFR & 8.3 & 4.5 & 5.2 & 5.4 & 18.5 & 5.6 & 3.1 & 4.1 & 3.0 & 19.2 \\
\hline RFL & 7.3 & 6.1 & 2.9 & 4.7 & 17.3 & 5.9 & 6.7 & 5.3 & 4.5 & 17.4 \\
\hline RFR & 9.9 & 6.6 & 9.1 & 5.2 & 25.9 & 5.1 & 5.7 & 8.1 & 5.0 & 22.3 \\
\hline SML & 7.9 & 6.7 & 5.6 & 6.5 & 10.2 & 5.5 & 4.7 & 5.4 & 4.5 & 14.1 \\
\hline SMR & 4.1 & 5.6 & 5.0 & 4.5 & 8.7 & 3.4 & 4.0 & 4.9 & 3.5 & 9.9 \\
\hline STL & 5.7 & 4.6 & 5.0 & 4.3 & 10.2 & 5.1 & 6.5 & 4.7 & 4.8 & 9.3 \\
\hline STR & 6.2 & 5.1 & 6.7 & 5.8 & 6.8 & 5.5 & 5.1 & 7.0 & 5.5 & 8.7 \\
\hline VIL & 7.7 & 6.1 & 4.8 & 5.9 & 9.8 & 5.8 & 6.6 & 7.0 & 5.9 & 13.6 \\
\hline VIR & 13.5 & 5.2 & 4.1 & 8.1 & 8.9 & 4.7 & 2.5 & 2.3 & 2.9 & 12 \\
\hline VLL & 5.8 & 5.9 & 6.9 & 5.6 & 12.6 & 5.5 & 6.3 & 7.1 & 5.6 & 13.2 \\
\hline VLR & 8.0 & 7.5 & 10.2 & 7.7 & 32.3 & 7.7 & 7.9 & 11.8 & 7.8 & 37.7 \\
\hline VML & 7.2 & 4.8 & 4.3 & 5.4 & 6.8 & 3.6 & 2.8 & 2.2 & 2.6 & 6.9 \\
\hline VMR & 4.7 & 4.3 & 4.9 & 4.5 & 7.3 & 2.7 & 3.1 & 4.4 & 3.1 & 8.1 \\
\hline
\end{tabular}

$R F R$, right rectus femoris; $R F L$, left rectus femoris; VMR, right vastus medialis; VML, left vastus medialis; VIR, right vastus intermedius; VIL, left vastus intermedius; $V L R$, right vastus lateralis; $V L L$, left vastus lateralis; $S T R$, right semitendinosus; $S T L$, left semitendinosus; $B F R$, right biceps femoris long head; $B F L$, left biceps femoris long head; $S M R$, and right semimembranosus; $S M L$, left semimembranosus. *Significant difference $p<0.05$ caused by direct saturation of water, as this lipid resonance is very close to the water peak.

$\mathrm{B}^{+}$inhomogeneities due to constructive and destructive interference of the transmit field are a common problem at ultrahigh fields $\left(\geq 7 \mathrm{~T}\right.$ ). However, $\mathrm{B}^{+}$inhomogeneities can also lead to regional loss of signal (shading artifacts) at $3 \mathrm{~T}$ in particular in the thighs, hips, and pelvis region. In this work, we employed dielectric pads to locally augment the transmit field and reduce the shading artifacts. Although in many cases the shading was still slightly visible, the pads were capable of recovering most of the signal as evidenced from similar high SNR levels for both the right $(41.8 \pm 16.0)$ and left $(41.7 \pm$ 17.1) legs.

To investigate whether we could further optimize the transmit field homogeneity, we performed additional manual $\mathrm{B}^{+}$ calibration prior to the DTI sequence for five subjects. This

Table 4 Mean wsCV and standard deviation (\%) per DTI parameter

\begin{tabular}{lcc}
\hline DTI parameter & IVIM & Standard \\
\hline MD & $4.5 \pm 1.4^{*}$ & $5.6 \pm 1.2^{*}$ \\
FA & $15.2 \pm 8.0$ & $13.9 \pm 7.8$ \\
$\lambda_{1}$ & $5.3 \pm 1.5^{*}$ & $7.5 \pm 2.4^{*}$ \\
$\lambda_{2}$ & $4.9 \pm 1.8$ & $5.5 \pm 1.1$ \\
$\lambda_{3}$ & $5.7 \pm 2.5$ & $5.7 \pm 2.0$ \\
\hline
\end{tabular}

*Significant difference $p<0.05$ further improved signal homogeneity, reduced the fat and chemical shift artifacts, and increased SNR with fewer muscles excluded, i.e., 2 out of 140 muscles versus 48 out of 280 muscles. Importantly, the wsCV values per DTI parameter were lower, suggesting higher repeatability. This is also in line with the simulation work of Damon [16]. Additionally, Filli et al performed twofold and threefold acceleration of the DTI sequence using simultaneous multi-slice acquisition, with the twofold acceleration achieving similar fibertracking and quantitative results as the conventional DTI sequence [27]. This could decrease the scanning time and further increase the clinical applicability of this protocol.

\section{DTI parameters}

The muscle DTI parameter values were comparable with those reported in previous studies [28, 29]. IVIM correction reduced all parameter values except FA. This is in line with previous work in the human calf, which showed that not accounting for perfusion leads to MD and eigenvalue overestimation and FA underestimation [20].

We found that the wsCV values for the three eigenvalues and MD were on average lower after IVIM modeling. In contrast, wsCV values for FA were on average higher after IVIM correction. A similar pattern was observed when comparing our IVIM-corrected diffusion parameters to previous work in forearm muscles [28]. Furthermore, the minimal detectable difference values for both the eigenvalues and MD were lower 

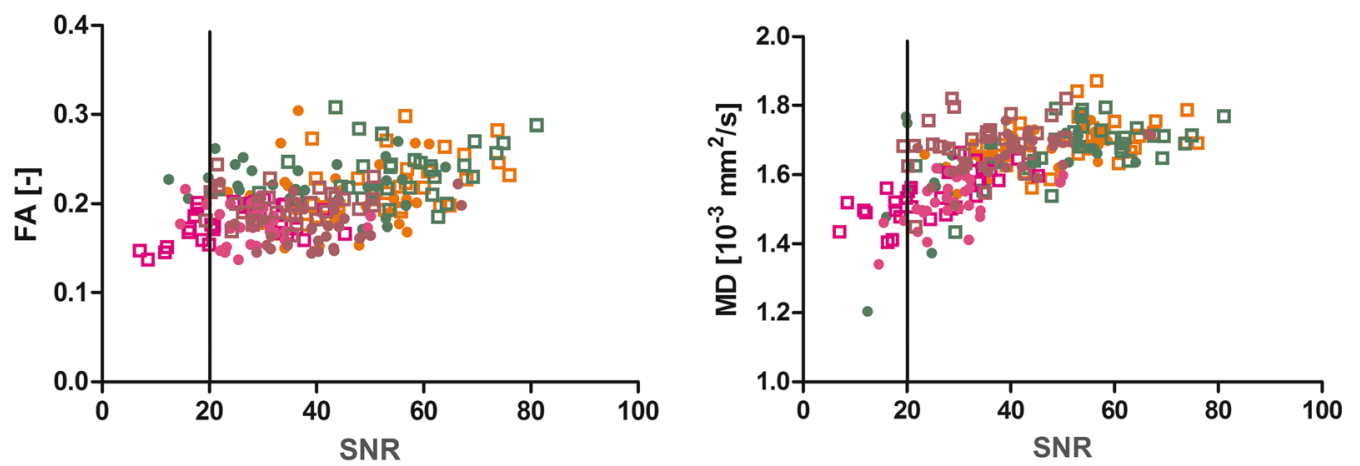

Left Biceps Femoris

- Right Biceps Femoris

- Left Semitendinosus

- Right Semitendinosus

- Left Vastus Medialis

- Right Vastus Medialis

- Left Vastus Intermedius

- Right Vastus Intermedius

Fig. 6 IVIM-corrected FA (top graph) and MD (bottom graph) as function of SNR for each individual muscle

for IVIM-corrected DTI data compared with those for standard analysis. Taken these findings combined suggests that IVIM modeling positively affects repeatability of the diffusion parameters with exception of FA and as a result improves the detection limit of the technique. Previous research has shown that changes in DTI parameters after exercise and/or injury are mostly present in MD, $\lambda_{2}$, and $\lambda_{3}$ (radial diffusivity) [11, 30, 31]. Therefore, achieving high repeatability for MD, RD, and the eigenvalues is essential. Hence, we recommend the use of IVIM correction for its positive effect on the repeatability of MD and the eigenvalues. Importantly, DTI parameters for the left and right legs had similar repeatability, indicating that bilateral imaging protocol used here can accurately quantify diffusion parameters in both legs simultaneously and the contralateral leg thus can be used as internal control.

\section{Study limitations}

Some limitations of the study should be acknowledged. Firstly, we included 13 males and only 2 females. This was done to reflect the demographics of our hamstring injury patients, which are predominantly male soccer players. Men have a higher incidence rate of hamstring injuries compared with women, even when practicing the same sport [32]. Secondly, our subjects were not rested for $30 \mathrm{~min}$ prior to scanning as was previously recommended [33], which may have increased the variance. Furthermore, we did not collect data on the time interval between the last exercise session of the subjects and the MRI examinations. Exercise and postexercise muscle edema might have caused further variance in DTI parameters [30].

Additionally, SNR values were estimated from the PCA denoising and not from a separate noise map acquisition. This may have resulted in a systemic underestimation of the SNR and exclusion of some datasets based on the strict SNR threshold of 20. Better SNR estimates would improve the repeatability of the DTI parameters due to the known correlation between SNR and repeatability [16]. Furthermore, an additional $\mathrm{B} 1^{+}$calibration was only performed for 5 subjects. We believe that repeatability would have been even higher if this additional $\mathrm{B}^{+}$calibration had been performed for all subjects.

\section{Conclusion}

DTI parameters (MD and eigenvalues), except FA, were repeatable. IVIM correction and additional $\mathrm{B}^{+}$calibration further improved the repeatability. On average, wsCV values were slightly lower than the values reported in previous studies. We plan to use this protocol in future studies on subjects with leg muscle injury.

Funding This work was supported by a Sportinnovator grant of The Netherlands Organization for Health Research and Development, ZonMw (grant number: 50-53800-98-PR020).

\section{Compliance with ethical standards}

Guarantor The scientific guarantor of this publication is Aart Nederveen.

Conflict of interest The authors of this manuscript declare no relationships with any companies, whose products or services may be related to the subject matter of the article.

Statistics and biometry Jonáh Stunt kindly provided statistical advice for this manuscript.

Informed consent Written informed consent was obtained from all subjects in this study.

Ethical approval Institutional Review Board approval was not required because no patients were enrolled and no medical questions were answered.

Study subjects or cohorts overlap Ten study subjects have been previously reported in an E-poster presentation at the ISMRM joint annual meeting 2018 in Paris.

\footnotetext{
Methodology

- prospective

- diagnostic or prognostic study

- performed at one institution
} 
Open Access This article is distributed under the terms of the Creative Commons Attribution 4.0 International License (http:// creativecommons.org/licenses/by/4.0/), which permits unrestricted use, distribution, and reproduction in any medium, provided you give appropriate credit to the original author(s) and the source, provide a link to the Creative Commons license, and indicate if changes were made.

\section{References}

1. Järvinen TA, Järvinen TL, Kääriäinen $M$, Kalimo $H$, Järvinen $M$ (2005) Muscle injuries: biology and treatment. Am J Sports Med 33:745-764

2. Wangensteen A, Tol JL, Witvrouw E et al (2016) Hamstring reinjuries occur at the same location and early after return to sport: a descriptive study of MRI-confirmed reinjuries. Am J Sports Med 44:2112-2121

3. Ekstrand J, Healy JC, Waldén M, Lee JC, English B, Hägglund M (2012) Hamstring muscle injuries in professional football: the correlation of MRI findings with return to play. Br J Sports Med 46: $112-117$

4. Greenky M, Cohen S (2017) Magnetic resonance imaging for assessing hamstring injuries: clinical benefits and pitfalls - a review of the current literature. Open Access J Sports Med 8:167-170

5. Reurink G, Goudswaard GJ, Tol JL et al (2014) MRI observations at return to play of clinically recovered hamstring injuries. Br J Sports Med 48:1370-1376

6. Reurink G, Whiteley R, Tol JL (2015) Hamstring injuries and predicting return to play: "bye-bye MRI?". Br J Sports Med 49: 1162-1163

7. Moen MH, Reurink G, Weir A, Tol JL, Maas M, Goudswaard GJ (2014) Predicting return to play after hamstring injuries. Br J Sports Med 48:1358-1363

8. Wangensteen A, Almusa E, Boukarroum S et al (2015) MRI does not add value over and above patient history and clinical examination in predicting time to return to sport after acute hamstring injuries: a prospective cohort of 180 male athletes. Br J Sports Med 49: $1579-1587$

9. Oudeman J, Nederveen AJ, Strijkers GJ, Maas M, Luijten PR, Froeling M (2016) Techniques and applications of skeletal muscle diffusion tensor imaging: a review. J Magn Reson Imaging 43:773788

10. Froeling M, Oudeman J, Strijkers GJ et al (2014) Muscle changes detected by diffusion-tensor imaging after long-distance running. Radiology 274:140702

11. Giraudo C, Motyka S, Weber M et al (2018) Normalized STEAMbased diffusion tensor imaging provides a robust assessment of muscle tears in football players: preliminary results of a new approach to evaluate muscle injuries. Eur Radiol 28:2882-2889

12. Fouré A, Pini L, Rappacchi S et al (2019) Ultrahigh-field multimodal MRI assessment of muscle damage. J Magn Reson Imaging 49:904-906

13. Brink WM, Versluis MJ, Peeters JM, Börnert P, Webb AG (2016) Passive radiofrequency shimming in the thighs at 3 Tesla using high permittivity materials and body coil receive uniformity correction. Magn Reson Med 76:1951-1956

14. Galbán CJ, Maderwald S, Uffmann K, Ladd ME (2005) A diffusion tensor imaging analysis of gender differences in water diffusivity within human skeletal muscle. NMR Biomed 18:489-498

15. Yanagisawa O, Shimao D, Maruyama K, Nielsen M, Irie T, Niitsu M (2009) Diffusion-weighted magnetic resonance imaging of human skeletal muscles: gender-, age- and muscle-related differences in apparent diffusion coefficient. Magn Reson Imaging 27:69-78
16. Damon BM (2008) Effects of image noise in muscle diffusion tensor (DT)-MRI assessed using numerical simulations. Magn Reson Med 60:934-944

17. Froeling M, Nederveen AJ, Nicolay K, Strijkers GJ (2013) DTI of human skeletal muscle: the effects of diffusion encoding parameters, signal-to-noise ratio and $\mathrm{T} 2$ on tensor indices and fiber tracts. NMR Biomed 26:1339-1352

18. Koh D-M, Collins DJ, Orton MR (2011) Intravoxel incoherent motion in body diffusion-weighted MRI: reality and challenges. JR Am J Roentgenol 196:1351-1361

19. Iima M, Le Bihan D (2015) Clinical intravoxel incoherent motion and diffusion MR imaging: past, present, and future. Radiology 278:13-32

20. De Luca A, Bertoldo A, Froeling M (2017) Effects of perfusion on DTI and DKI estimates in the skeletal muscle. Magn Reson Med 78:233-246

21. Brink WM, Gulani V, Webb AG (2015) Clinical applications of dual-channel transmit MRI: a review. J Magn Reson Imaging 42: 855-869

22. Veraart J, Novikov DS, Christiaens D, Ades-Aron B, Sijbers J, Fieremans E (2016) Denoising of diffusion MRI using random matrix theory. Neuroimage 142:394-406

23. Klein S, Staring M, Pluim JP (2007) Evaluation of optimization methods for nonrigid medical image registration using mutual information and B-splines. IEEE Trans Image Process 16. https://doi. org/10.1109/TIP.2007.909412

24. Veraart J, Sijbers J, Sunaert S, Leemans A, Jeurissen B (2013) Weighted linear least squares estimation of diffusion MRI parameters: strengths, limitations, and pitfalls. Neuroimage 81:335-346

25. Veraart J, Fieremans E, Novikov DS (2016) Diffusion MRI noise mapping using random matrix theory. Magn Reson Med 76:15821593

26. Yushkevich PA, Piven J, Hazlett HC et al (2006) User-guided 3D active contour segmentation of anatomical structures: significantly improved efficiency and reliability. Neuroimage 31:1116-1128

27. Filli L, Piccirelli M, Kenkel D, et al (2015) Simultaneous multislice echo planar imaging with blipped controlled aliasing in parallel imaging results in higher acceleration: A promising technique for accelerated diffusion tensor imaging of skeletal muscle. Invest Radiol 50:456-463

28. Froeling M, Oudeman J, van den Berg S et al (2010) Reproducibility of diffusion tensor imaging in human forearm muscles at 3.0 T in a clinical setting. Magn Reson Med 64:11821190

29. Sinha S, Sinha U (2011) Reproducibility analysis of diffusion tensor indices and fiber architecture of human calf muscles in vivo at 1.5 Tesla in neutral and plantarflexed ankle positions at rest. J Magn Reson Imaging 34:107-119

30. Cermak NM, Noseworthy MD, Bourgeois JM, Tarnopolsky MA, Gibala MJ (2012) Diffusion tensor MRI to assess skeletal muscle disruption following eccentric exercise. Muscle Nerve 46:42-50

31. Bruschetta D, Anastasi G, Andronaco V et al (2018) Human calf muscles changes after strength training as revealed by diffusion tensor imaging. J Sports Med Phys Fitness 59. https://doi.org/10. 23736/S0022-4707.18.08759-5

32. Cross KM, Gurka KK, Saliba S, Conaway M, Hertel J (2013) Comparison of hamstring strain injury rates between male and female intercollegiate soccer athletes. Am J Sports Med 41:742-748

33. Elzibak AH, Noseworthy MD (2014) Assessment of diffusion tensor imaging indices in calf muscles following postural change from standing to supine position. MAGMA 27:387-395

Publisher's note Springer Nature remains neutral with regard to jurisdictional claims in published maps and institutional affiliations. 